\title{
Aggressive sebaceous carcinoma of the scalp: a case report and literature review
}

\author{
Guihong Li ${ }^{1}$, Jing Shen ${ }^{2}$, Haiyan Huang ${ }^{3}$, Ruili Chen ${ }^{4}$, Guangtong Zhou ${ }^{1}$ \\ ${ }^{1}$ Department of Neurosurgery, The First Affiliated Hospital of Zhengzhou University, Zhengzhou, China; ${ }^{2}$ Department of Cardiopulmonary \\ Function, Henan Provincial Chest Hospital, Zhengzhou, China; ${ }^{3}$ Department of Neurosurgery, The First Hospital of Jilin University, Changchun, \\ China; ${ }^{4}$ Department of Dermatology, The First Hospital of Jilin University, Changchun, China \\ Correspondence to: Guihong Li. Department of Neurosurgery, The First Affiliated Hospital of Zhengzhou University, Zhengzhou 450000, China. \\ Email: lighns@hotmail.com.
}

\begin{abstract}
Sebaceous carcinoma (SC) is an uncommon and potentially aggressive adnexal neoplasm. SC presents most often as a periocular tumor arising from the ocular adnexa with unclear pathogenesis. Aggressive SC of the scalp is extremely rare. Here, we describe a rare case of aggressive SC of the scalp in a 61-year-old female, who presented with a scalp neoplasm lasting for two months. Cranial magnetic resonance imaging (MRI) revealed a partly necrotic and cystic tumor with mixed signal shadow. The parietal multiple destructive lesions invaded the skull, involved the dura mater, and compressed the associated superior sagittal sinus. A wide local lesion excision with I stage repair of dura mater and I stage bone graft of skull was performed in our department. The patient was treated with external radiation to prevent recurrence and was followed for 3 years with favorable results. The relevant literature regarding aggressive SC was reviewed, and the clinical manifestations, radiological characteristics, surgical strategies, histopathological findings, and prognosis are discussed. This cutaneous malignancy invaded skull, dura mater or brain, or metastasized to the lymph nodes and viscera, with high recurrence and fatality rates. It is necessary to be aware of these rare examples which showed unexpectedly clinical behaviors. Early precise diagnosis and improved radical treatment remain essential steps against aggressive cutaneous malignancy. Patients with aggressive SC of the scalp should be closely followed to assess recurrence and distant metastasis.
\end{abstract}

Keywords: Scalp; aggressive; sebaceous carcinoma (SC); case report

Submitted Dec 16, 2020. Accepted for publication Jul 16, 2021.

doi: $10.21037 /$ tcr-20-3443

View this article at: https://dx.doi.org/10.21037/tcr-20-3443

\section{Introduction}

Sebaceous carcinoma (SC) is a rare and potentially aggressive adnexal neoplasm that was first described by Straatsma in 1956 (1). SC most commonly presents as a periocular tumor arising from the ocular adnexa (2). Extraocular SC is considered as a less aggressive neoplasm and occurs most commonly on the neck and scalp (3). The pathogenesis of SC is poorly understood. However, MuirTorre syndrome (MTS), a genetic disease associated with visceral neoplasms is significantly associated with it (4). Aggressive SC of the scalp is extremely rare, and only five previous cases having been found in the literature (5-9). These cutaneous malignancies invaded skull, dura mater, brain, or metastasized to the lymph nodes and viscera, with high recurrence and fatality rates. Herein, an aggressive SC of the scalp is presented that involved dura mater, and the clinical profiles from relevant literature are summarized.

We present the following article in accordance with the CARE reporting checklist (available at https://dx.doi. org/10.21037/tcr-20-3443).

\section{Case presentation}

A 61-year-old female was admitted to The First Hospital of Jilin University (Changchun, Jilin, China), after presenting with a scalp neoplasm lasting for two months. Physical 

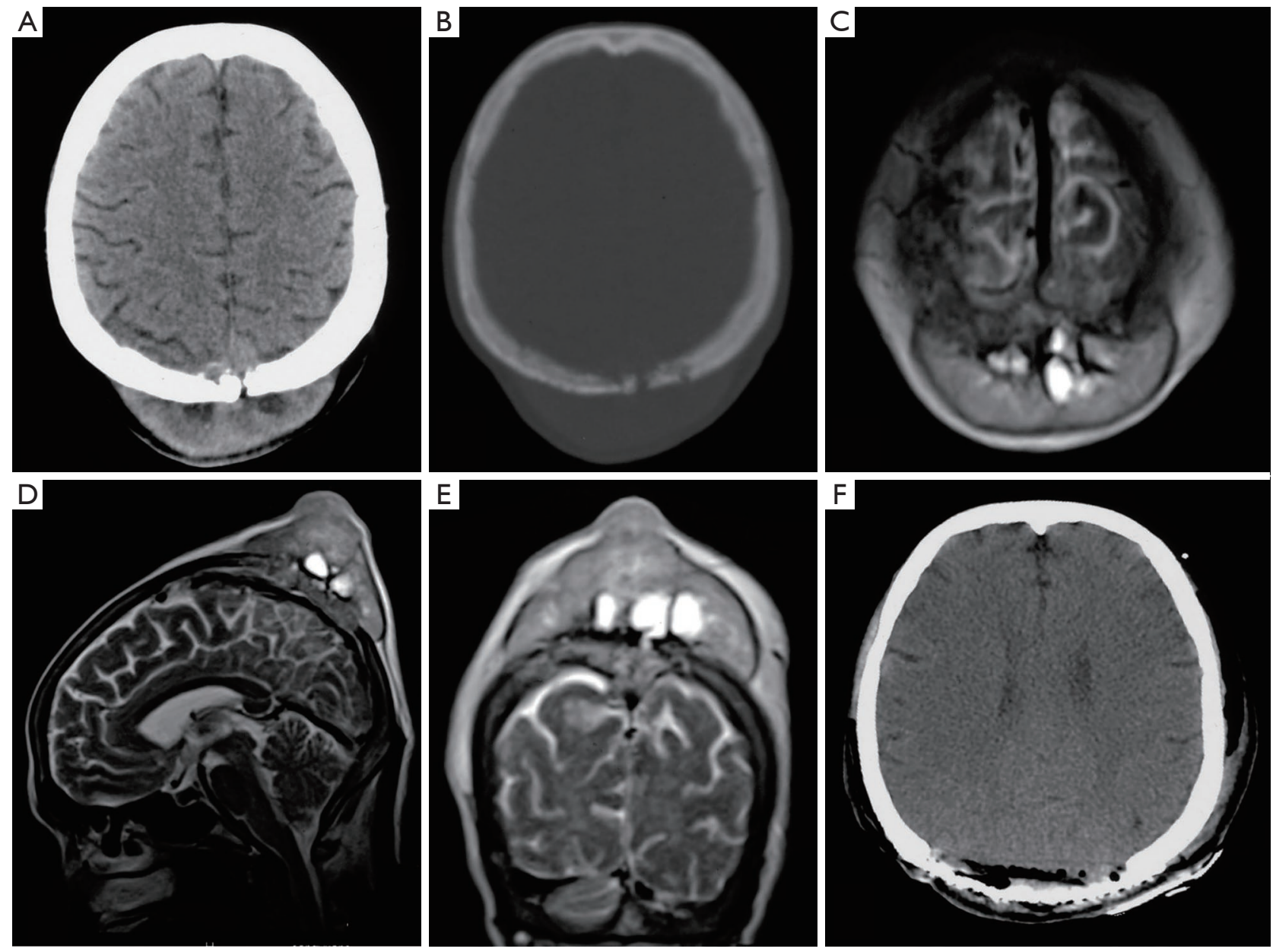

Figure 1 Preoperative examination and postoperative CT. (A) CT of head showed a mixed density shadow in the subcutaneous soft tissue of the bilateral parietal; (B) bone window CT showed that the parietal bone density was significantly uneven with bone discontinuity; (C,D,E) cranial MRI revealed a partly necrotic and cystic tumor with mixed signal shadow in T2WI. The parietal multiple destructive lesions Invaded the skull, involving the dura mater, and compressed the associated superior sagittal sinus. (C) axial T2WI; (D) sagittal T2WI; (E) coronal T2WI; (F) postoperative CT confirmed that the tumor was completely removed, and the skull was repaired with the titanium plate bone graft. CT, computed tomography; MRI, magnetic resonance imaging.

examination revealed a $6 \mathrm{~cm}$ mass across the parietal region of her scalp, which was oval, markedly tender, without pus rupture, and fixed to the skull. There was no neurological deficit or swollen lymph node. There was no other neoplasm presented in the patient or her family.

Computed tomography (CT) showed a mixed density shadow, approximately $7.5 \mathrm{~cm} \times 4 \mathrm{~cm}$, in the subcutaneous soft tissue of the bilateral parietal (Figure 1A). The CT in the bone window showed that the parietal bone was discontinuous and the density was significantly uneven (Figure $1 B$ ). Cranial magnetic resonance imaging (MRI) revealed a partly necrotic and cystic tumor with mixed signal shadow in T2WI. The parietal multiple destructive lesions invaded the skull, involved the dura mater, and compressed the associated superior sagittal sinus (Figure $1 C, D, E$ ). The patient was diagnosed with a subcutaneous mass that tended to be malignant.

A wide local lesion excision with I stage repair of dura mater and I stage bone graft of skull was performed in our department. The scalp was cut along the anterior and posterior side of the tumor with a spindle shaped incision, and then the blood-rich cystic tumor tissue was found under the scalp. The tumor eroded, damaged the skull, and adhered tightly to the dura mater, but did not invade the 

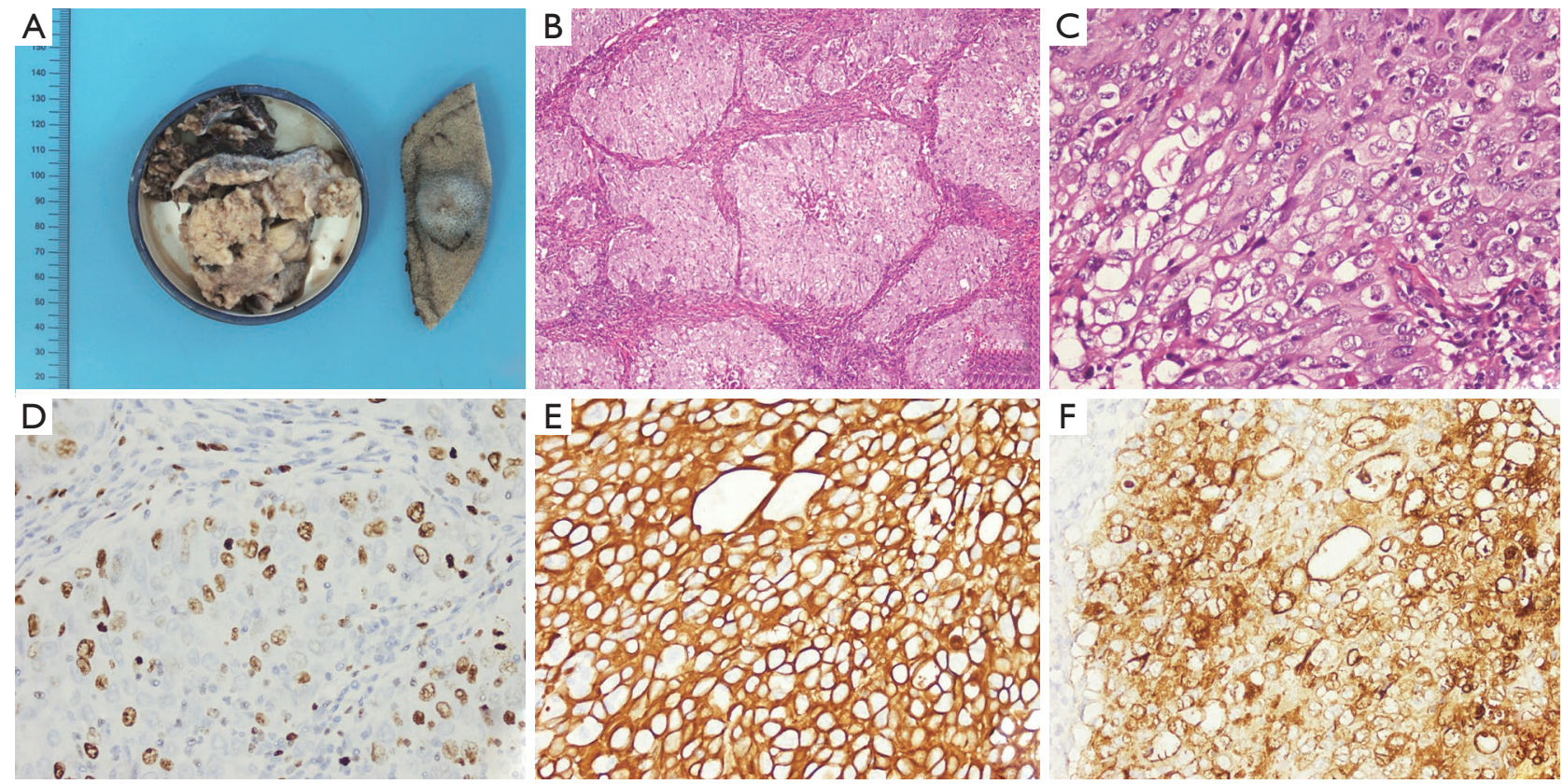

Figure 2 General photo and the histological findings of the tumor. (A) General photo of the pathology after surgery showing the excised scalp, tumor, and part of the skull; (B) paraffin-embedded hematoxylin and eosin stained sections show unencapsulated, lobular, dermally based collections of sebaceous, and the presence of atypical mitoses, necrosis and vascular invasion (H\&E staining, $\times 100)$; (C) these lobules consisted of atypic pleomorphic cells containing nuclear pleomorphism, nuclear hyperchromatism, mitotic figures, and foamy vacuolization of the cytoplasm (H\&E staining, $\times 400)$; (D,E,F) immunohistochemical staining was positive for Ki-67 (D, ×400), CK-pan (E, $\times 400)$, and $\operatorname{EMA}(\mathrm{F}, \times 400)$. EMA, epithelial membrane antigen.

brain tissue. After the tumor was completely resected, the dura was repaired with artificial dura mater and repaired the skull with titanium plate bone graft (Figure $1 F)$.

The surgically resected tumor tissue was available for histopathological examination after photographing (Figure 2A). Paraffin-embedded hematoxylin and eosin (H\&E) stained sections showed unencapsulated, lobular, dermally based collections of sebaceous, and the present of atypical mitoses, necrosis and vascular invasion (Figure 2B). These lobules consisted of atypic pleomorphic cells containing nuclear pleomorphism, nuclear hyperchromatism, mitotic figures, and foamy vacuolization of the cytoplasm (Figure 2C). Immunohistochemical staining (Figure 2D,E,F) was positive for Ki-67, CK-pan, and epithelial membrane antigen (EMA). After operation, the patient was then treated with radiation. The radiotherapy modality was conventional postoperative electronic radiation therapy, the irradiation range was the operation area, and the radiation dose was $60 \mathrm{~Gy} / 2 \mathrm{~Gy} / 30 \mathrm{f}$. The patient was followed for 3 years and the results have been favorable. And the patient praised our diagnosis and treatment.
All procedures performed in studies involving human participants were in accordance with the ethical standards of the institutional and/or national research committee(s) and with the Helsinki Declaration (as revised in 2013). Written informed consent was obtained from the patient.

\section{Discussion}

The MEDLINE database was searched for articles related to aggressive SC of the scalp published from 1945 to 2020. A total of five publications reporting on five patients were retrieved. The clinical profiles are summarized in Table 1.

Up to $75 \%$ of all SC presents as a periocular tumor arising from the ocular adnexa (10). Aggressive SC of the scalp is extremely rare. A total of five cases of locally aggressive SC of scalp were found in the literature, of which three were aggressive to the brain, one invaded the dura mater, one invaded the skull, and three distantly metastasized to the lymph nodes or viscera. The present case invaded the dura mater and did not metastasize. Multiple studies have identified a female predominance among patients diagnosed 


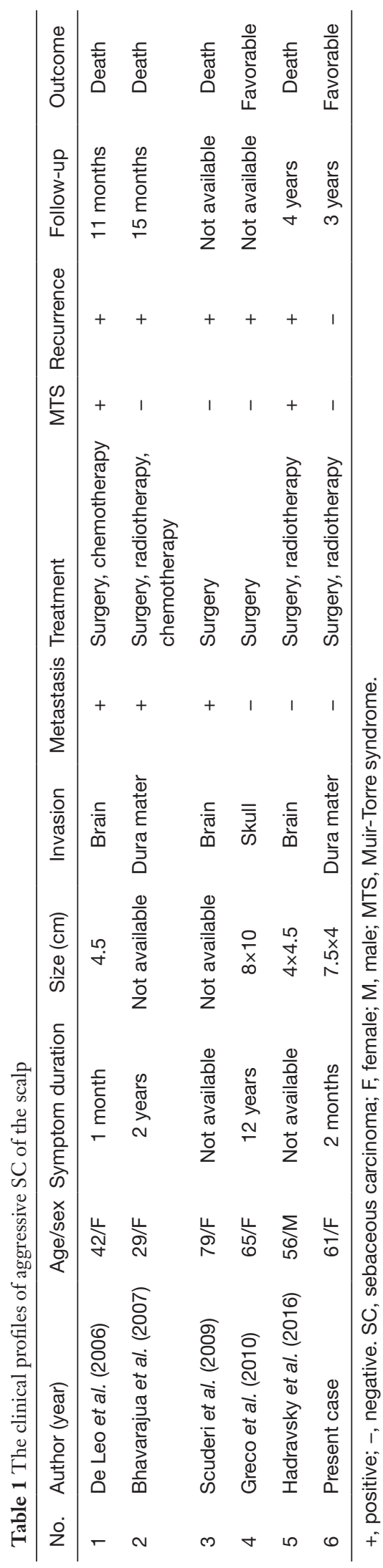

with SC $(11,12)$. Including this case, a total of six patients are reported, of whom five were females, accounting for $83.3 \%$, and the average age was 55 years old, which was consistent with the characteristics of previous studies. Extraocular SC commonly presents as a painless papule, or cystic nodule, which grows rapidly (13). Regardless of the duration of the symptoms, all the patients retrieved had the characteristics of rapid enlargement in lesions. The importance of a thorough physical examination cannot be emphasized enough, and early detection is critical to provide patients with the best chance for a favorable outcome (14). Extraocular SC can occur sporadically or in the setting of MTS (4). Of the five retrieved cases, two were related to MTS and both had poor prognosis, but our current case did not. Therefore, it is import to acquire the patients' medical history about neoplasm. In addition, imaging inspection appears to be crucial for aggressive SC of the scalp. CT may reveal skull destruction, and MRI can delineate intracranial soft tissue involvement by tumor. Biopsy of tumor is essential for diagnosis.

Sebaceous tumors are separated into three major categories: sebaceous adenoma, basal cell carcinoma with sebaceous differentiation, and SC (13). Four histologic patterns of SC are commonly recognized: lobular, comedocarcinoma, papillary, and mixed. Examination of routine $\mathrm{H} \& \mathrm{E}$ paraffin embedded permanent sections demonstrates lobular architecture, high mitotic activity, nuclear pleomorphism, and foamy vacuolization of the cytoplasm $(12,15)$. Lipids present in the cytoplasm of the cells result in their characteristic "foamy", which stain positive with oil red $\mathrm{O}$ and Sudan black in fresh-frozen samples (12). Immunohistochemistry has largely replaced lipid stains such as oil red $\mathrm{O}$, of which the sensitivity decreases after formalin fixation, in the diagnosis of SC (15). Extraocular SC cells stain with EMA, cytokeratin (CK), Ber-EP4, and androgen receptor (13). Compared to benign counterparts, extraocular SC expresses increased p53 and Ki-67 levels (13). In the current case, Ki-67, CK-pan, and EMA are highly expressed, which is consistent with the typical immunohistochemical features of SC. The main differential diagnoses are clear cell hidradenocarcinoma, clear cell melanoma, squamous cell carcinoma composed of clear cells, metastatic renal cell carcinoma, and metastatic prostate carcinoma.

Mohs micrographic surgery (MMS) with lower local recurrence rates is the best initial treatment modality for SC (12), especially those with lesions in cosmetically sensitive areas. However, aggressive SC of the scalp 
has very poor prognosis with $83.3 \%$ recurrence rate. Therefore, it is recommended that an enlarged resection be used for the intracranial invasive tumor and then repair of the dura mater or skull in I stage. The extensive scalp defects can be reconstructed using large local scalp flap transposition $(16,17)$. Wide resection with craniectomy and reconstruction with microvascular free tissue transfer provides safe and reliable treatment of recalcitrant invasive scalp skin cancers (18). The role of radiation therapy and systemic chemotherapy after surgery is not clear in the extraocular SC, which are only suggested for patients who are poor surgical candidates or those with recurrent or metastatic disease $(12,13,19)$. Electrochemotherapy has shown to be an interesting tool for treatment of metastatic extraocular SC (20). The patient was treated with external radiation to prevent recurrence and was followed for 3 years with favorable results.

\section{Conclusions}

In conclusion, an extremely rare case of aggressive $\mathrm{SC}$ on the scalp is reported here. It is necessary to be aware of these rare examples which showed unexpectedly clinical behaviors. Early precise diagnosis and improved radical treatment remain an essential step against aggressive cutaneous malignancy. Patients with aggressive SC of the scalp should be followed up closely to assess recurrence and distant metastasis.

\section{Acknowledgments}

Funding: None.

\section{Footnote}

Reporting Checklist: The authors have completed the CARE reporting checklist. Available at https://dx.doi. org/10.21037/tcr-20-3443

Conflicts of Interest: All authors have completed the ICMJE uniform disclosure form (available at https://dx.doi. org/10.21037/tcr-20-3443). The authors have no conflicts of interest to declare.

Ethical Statement: The authors are accountable for all aspects of the work in ensuring that questions related to the accuracy or integrity of any part of the work are appropriately investigated and resolved. All procedures performed in studies involving human participants were in accordance with the ethical standards of the institutional and/or national research committee(s) and with the Helsinki Declaration (as revised in 2013). Written informed consent was obtained from the patient for publication of this case report and accompanying images. A copy of the written consent is available for review by the editorial office of this journal.

Open Access Statement: This is an Open Access article distributed in accordance with the Creative Commons Attribution-NonCommercial-NoDerivs 4.0 International License (CC BY-NC-ND 4.0), which permits the noncommercial replication and distribution of the article with the strict proviso that no changes or edits are made and the original work is properly cited (including links to both the formal publication through the relevant DOI and the license). See: https://creativecommons.org/licenses/by-nc-nd/4.0/.

\section{References}

1. Straatsma BR. Meibomian gland tumors. AMA Arch Ophthalmol 1956;56:71-93.

2. Urban FH, Winkelmann RK. Sebaceous malignancy. Arch Dermatol 1961;84:63-72.

3. Bassetto F, Baraziol R, Sottosanti MV, et al. Biological behavior of the sebaceous carcinoma of the head. Dermatol Surg 2004;30:472-6.

4. Muir EG, Bell AJ, Barlow KA. Multiple primary carcinomata of the colon, duodenum, and larynx associated with kerato-acanthomata of the face. Br J Surg 1967;54:191-5.

5. De Leo A, Innocenzi D, Onesti MG, et al. Extraocular sebaceous carcinoma in Muirr Torre Syndrome with unfavorable prognosis. Cancer Chemother Pharmacol 2006;58:842-4.

6. Bhavarajua VM, Shamim SE, Naik VR, et al. Sebaceous cell carcinoma of scalp - a rare presentation. Malays J Med Sci 2007;14:67-70.

7. Scuderi S, Giaccone M, D'Errico E, et al. Aggressive sebaceous carcinoma of the head. Chir Ital 2009;61:401-4.

8. Greco M, Vitagliano T, Fiorillo MA, et al. Rare malignant tumors of the scalp: a report of four cases, their treatment and a review of the literature. Eur Rev Med Pharmacol Sci 2010;14:993-7.

9. Hadravsky L, Kazakov DV, Stehlik J, et al. Aggressive Extraocular Sebaceous Carcinoma of the Scalp Involving the Brain in a Patient With Muir-Torre Syndrome. Am J 
Dermatopathol 2016;38:618-22.

10. Rao NA, Hidayat AA, McLean IW, et al. Sebaceous carcinomas of the ocular adnexa: A clinicopathologic study of 104 cases, with five-year follow-up data. Hum Pathol 1982;13:113-22.

11. Chikhalkar S, Garg G, Gutte R, et al. Sebaceous carcinoma of scalp with proliferating trichilemmal cyst. Indian Dermatol Online J 2012;3:138-40.

12. Kyllo RL, Brady KL, Hurst EA. Sebaceous carcinoma: review of the literature. Dermatol Surg 2015;41:1-15.

13. Knackstedt T, Samie FH. Sebaceous Carcinoma: A Review of the Scientific Literature. Curr Treat Options Oncol 2017;18:47.

14. Richmond HM, Duvic M, Macfarlane DF. Primary and metastatic malignant tumors of the scalp: an update. Am J Clin Dermatol 2010;11:233-46.

15. Nelson BR, Hamlet KR, Gillard M, et al. Sebaceous carcinoma. J Am Acad Dermatol 1995;33:1-15; quiz 16-8.

16. Wackym PA, Feuerman T, Strasnick B, et al.

Cite this article as: Li G, Shen J, Huang H, Chen R, Zhou G. Aggressive sebaceous carcinoma of the scalp: a case report and literature review. Transl Cancer Res 2021;10(9):4237-4242. doi: $10.21037 /$ tcr-20-3443
Reconstruction of massive defects of the scalp, cranium, and dura after resection of scalp neoplasms. Head Neck 1990;12:247-53.

17. Chao AH, Yu P, Skoracki RJ, et al. Microsurgical reconstruction of composite scalp and calvarial defects in patients with cancer: a 10-year experience. Head Neck 2012;34:1759-64.

18. Marijon P, Bertolus C, Foy JP, et al. Custom surgical management of invasive malignant tumors of the scalp. Acta Neurochir (Wien) 2020;162:2991-9.

19. Hu J, Xiao W, He Z, et al. Target splitting non-coplanar RapidArc radiation therapy for a diffuse sebaceous carcinoma of the scalp: a novel delivery technique. Radiat Oncol 2014;9:204.

20. Ribero S, Baduel ES, Brizio M, et al. Metastatic sebaceous cell carcinoma, review of the literature and use of electrochemotherapy as possible new treatment modality. Radiol Oncol 2016;50:308-12. 